\title{
ANALYSIS THE AGE CHARACTERISTICS OF BILIRUBIN ENCEPHALOPATHY IN
} NEONATAL

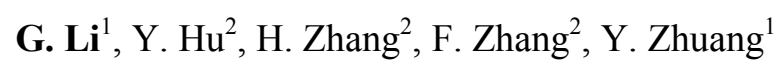 \\ ${ }^{1}$ Neonatology, ${ }^{2}$ Hunan Children's Hospital, Changsha, China
}

Objective: To investigate the age characteristics of bilirubin brain injury in neonatal.

Method: From November 2008 to October 2009, we collected 2252 cases which was diagnosed as neonatal bilirubinemia in our hospital. we divided all cases into $\sim 24$ hours, $\sim 72$ hours, $\sim 168$ hours, $\sim 336$ hours and $>$ 336 hours group, analysis the incidence of bilirubin encephalopathy .

Results: The incidence of bilirubin brain damage in $\sim 24$ hours group was highest and the incidence of bilirubin brain damage decreased with the increasing of age. However, according to the hour age of neonatal, the incidence of brain injury in $\sim 168$ hours group and $\sim 336$ hours group was highest, then followed by $>336$ hours group, $\sim 24$ hours group and 72 hours group got the lowest incidence. Depending on the time of jaundice duration before admission, the incidence of bilirubin brain injury in each group were: $7.43 \%$ (68/915), 14.07\% (95/675), 15.71\% (52/331), 11.11\% (22/198), $5.26 \%(7 / 133)$, the difference was statistically significant $\left(\chi^{2}=30.73, \mathrm{P}=0.000\right)$. This indicate that there is a close relationship between the incidence of bilirubin brain injury and the hour age of neonatal, the hour age of onset, the time of jaundice duration. Age at admission ranged from 3 to 14 days of the neonatal still need active treatment, while in the choice of major intervention measures (exchange transfusion therapy), clinicians should take into account the day of onset and duration of jaundice at the same time. 